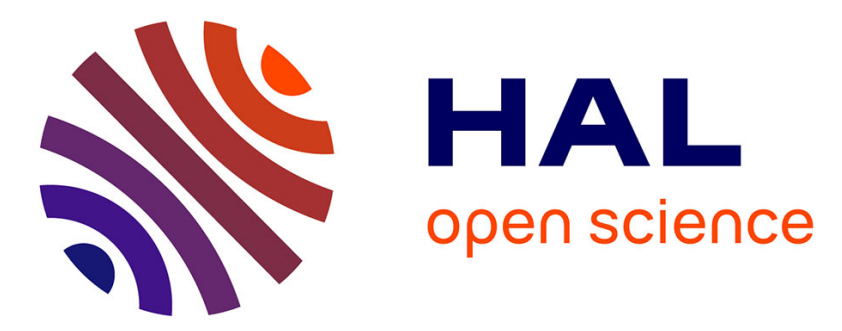

\title{
Adaptive shunted piezoelectric metacomposite: a new integrated technology for vibroacoustic control
}

Manuel Collet, Morvan Ouisse, Flaviano Tateo, Mohamed Ichchou, Tianli Huang

\section{- To cite this version:}

Manuel Collet, Morvan Ouisse, Flaviano Tateo, Mohamed Ichchou, Tianli Huang. Adaptive shunted piezoelectric metacomposite: a new integrated technology for vibroacoustic control. Workshop on Design, Control and Software Implementation for Distributed MEMS, Apr 2012, Besançon, France. 10.1109/dMEMS.2012.21 . hal-02300532

\section{HAL Id: hal-02300532 \\ https://hal.science/hal-02300532}

Submitted on 19 Nov 2019

HAL is a multi-disciplinary open access archive for the deposit and dissemination of scientific research documents, whether they are published or not. The documents may come from teaching and research institutions in France or abroad, or from public or private research centers.
L'archive ouverte pluridisciplinaire HAL, est destinée au dépôt et à la diffusion de documents scientifiques de niveau recherche, publiés ou non, émanant des établissements d'enseignement et de recherche français ou étrangers, des laboratoires publics ou privés. 


\section{Adaptive shunted piezoelectric metacomposite: a new integrated technology for vibroacoustic control}

\author{
M. Collet, M. Ouisse, F. Tateo \\ FEMTO-ST Dept Applied Mechanic \\ UMR CNRS 6174 \\ 24 rue de l'Épitaphe, 25000 Besançon, France \\ Email: manuel.collet@univ-fcomte.fr
}

\author{
M.N. Ichchou, T. Huang \\ LTDS, CNRS UMR5513 \\ Ecole Centrale de Lyon \\ 36 av Guy de Collongue, 69134 Ecully, France
}

\begin{abstract}
In this paper, we present an application of the Floquet-Bloch theorem in the context of electrodynamics for vibroacoustic power flow optimization by mean of distributed and shunted piezoelectric patches. The main purpose of this work is first to propose a dedicated numerical approach able to compute the multi-modal wave dispersions curves into the whole first Brillouin zone for periodically distributed 2D shunted piezomechanical systems. By using two specific indicators evaluating the evanescent part of Bloch's waves and the induced electronic damping, we optimize the piezoelectric shunting electrical impedance for controlling energy diffusion into the proposed semi-active distributed set of cells. Sound radiation efficiency is also analyzed for showing the effects of such smart metamaterial for controlling acoustical noise.

Index Terms-Distributed Active Noise Control, Adaptive
\end{abstract} Metacomposite, Distributed shunted piezoelectric patches

\section{INTRODUCTION}

Tailoring the dynamical behavior of wave-guide structures can provide an efficient and physically elegant means to optimize mechanical components with regards to vibration and acoustic criteria, among others. However, achieving this objective may lead to different outcomes depending on the context of the optimization. In the preliminary stages of a product's development, one mainly needs optimization tools capable of rapidly providing global design direction. Such optimization will also depend on the frequency range of interest. One usually discriminates between the low frequency (LF) range and the medium frequency (MF) range, especially if vibration and noise are considered. However, it should be noted that LF optimization of vibration is more common in the literature than MF optimization. For example, piezoelectric materials and other adaptive and smart systems are employed to improve the vibroacoustic quality of structural components, especially in the LF range [1], [2], [3]. Recently, much effort has been spent on developing new multi-functional structures integrating electro-mechanical systems in order to optimize their vibroacoustic behavior over a larger frequency band of interest[4], [5], [6], [7], [8], [9], [10], [11], [12]. However, there is still a lack of studies in the literature for MF optimization of structural vibration. To that end, the focus of this study is to provide a suitable numerical tool for computing wave dispersion in $2 \mathrm{D}$ periodic systems incorporating controlling electronics devices. The final aim is to allow their optimization in order to optimize vibroacoustic diffusion in 2D wave guides and analyze its effect on acoustic radiation

For optimizing wave dispersion we need to use efficient numerical tools. Two approaches can be distinguished for computing that dispersion: the semi-analytical finite element method (SAFE) and the wave finite element (WFE) method. In the former approach, the displacement field is modeled exactly in the direction of wave propagation by using a harmonic function and approximately in the perpendicular directions by using finite elements (FE). An eigenvalue problem is then formulated by introducing the displacement field into the governing equations. Solving the eigenvalue problem for a given frequency gives the wave numbers of all the propagating modes. The main disadvantage of the SAFE method is that FE used are not standard so they must be specifically defined for each application. Moreover the wave guide needs to be homogeneous. Nevertheless, a large amount of FE has been developed since 1975 to compute dispersion curves of rails [13], stiffened cylinders [14], laminated composite plates [15], [16] and viscoelastic laminated composite plates [17]. To avoid development of specific FE, the WFE method considers the structures as periodic in order to model a single period of the structure with standard FE. By using the periodic structure theory (PST) introduced by Mead [13], an eigenvalue problem can be formulated from the stiffness and mass matrices of the FE model to find wave numbers of all the propagating waves. Contrary to the SAFE method, the displacement field is now approximated in the direction of propagation. The WFE method has been successfully used to deal with wave propagation in two dimensional structures [18], [19]. The approach has also been used for fluid-structure systems using the theory of group representation for computing two dimensional dispersion in undamped vibroacoustic systems [20], [21]. One of the main problem of all these approaches is the difficulty to compute the damped wave numbers in the whole Brillouin domain of multiphysics problem incorporating shunted piezoelectric patches necessary for optimizing vibroacoustic behavior of such periodic smart structures [22], [23].

After recalling the Floquet-Bloch theorems, we introduce a new numerical formulation for computing the multi-modal damped wave numbers dispersion in the whole first Brillouin domain of a periodical smart structure made of periodically 
distributed shunted piezoelectric patches. Based on this wave modeling, optimization of the electrical impedance of the shunted circuit is performed in order to decrease the group velocity of flexural waves or to increase damping induced by the electric circuit. The obtained optimal impedances are also tested in controlling the MF response of a semi-distributed infinite system and its effect on sound radiation.

\section{Piezo-elasto-dynamical application of the FLOQUET-BLOCH THEOREM}

In this section the application of the celebrated FloquetBloch theorem is presented for piezo-elastodynamic problems. Based on the well known results obtained by Floquet [24] in one-dimensional and later rediscovered by Bloch [25] in multidimensional problems, we propose an original application to bi-dimensional piezo-elastodynamical problem leading to very general numerical implementation for computing waves dispersion for periodically smart distributed mechanical systems incorporating electronic components and damping effects [26].

\section{A. The Bloch Theorem}

The Bloch theorem gives the form of homogeneous states of Schrödinger equation with periodic potential. This theorem can be considered as a multidimensionnal application of the Floquet theorem [27]. The periodic medium properties satisfy a periodic condition as $M(\boldsymbol{x}+R \cdot \boldsymbol{m})=M(\boldsymbol{x}), \boldsymbol{m} \in \mathbb{Z}^{3}$ where $R=\left[\boldsymbol{r}_{1}, \boldsymbol{r}_{2}, \boldsymbol{r}_{3}\right] \in \mathbb{R}^{3 \times 3}$ is a matrix grouping the three lattice's basis vectors (in $3 \mathrm{D}$ ). We can also define the primitive cell as a convex polyhedron of $\mathbb{R}^{3}$ called $\Omega_{x}$. The reciprocal unit cell is denoted by $\Omega_{k}$ limited by the reciprocal lattice vector defined by the three vectors $\boldsymbol{g}_{j}$ so that: $\boldsymbol{r}_{i} \cdot \boldsymbol{g}_{j}=2 \pi \delta_{i j}$ ( $\delta_{i, j}$ being the Kronecker index). We note $G=\left[\boldsymbol{g}_{1}, \boldsymbol{g}_{2}, \boldsymbol{g}_{3}\right]$ the reciprocal lattice matrix in the later. If $\Omega_{x}$ is the irreductible primitive cell, $\Omega_{k}$ corresponds to the first Brillouin zone of the lattice. One can see [28] for details.

The Bloch Theorem stipulates that any functions $\boldsymbol{u}(\boldsymbol{x}) \in$ $L^{2}\left(\mathbb{R}^{3}, \mathbb{C}^{n}\right)$ can be expressed as

$$
\boldsymbol{u}(\boldsymbol{x})=\int_{\Omega_{k}} e^{i \boldsymbol{k} \boldsymbol{x}} \tilde{\boldsymbol{u}}(\boldsymbol{x}, \boldsymbol{k}) d \boldsymbol{k}
$$

where the Bloch amplitude $\tilde{\boldsymbol{u}}(\boldsymbol{x}, \boldsymbol{k})$ is $\Omega_{x}$-periodic and has the representations

$$
\begin{aligned}
\tilde{\boldsymbol{u}}(\boldsymbol{x}, \boldsymbol{k}) & =\sum_{\boldsymbol{n} \in \mathbb{Z}^{3}} \hat{\boldsymbol{u}}(\boldsymbol{k}+G \boldsymbol{n}) e^{i G \boldsymbol{n} \cdot \boldsymbol{x}} \\
\boldsymbol{u}(\boldsymbol{x}) & =\frac{\left|\Omega_{x}\right|}{(2 \pi)^{3}} \sum_{\boldsymbol{n} \in \mathbb{Z}^{3}} \boldsymbol{u}(\boldsymbol{x}+R \boldsymbol{n}) e^{i \boldsymbol{k}(\boldsymbol{x}+R \boldsymbol{n})}
\end{aligned}
$$

where $\hat{\boldsymbol{u}}(\boldsymbol{k})$ stands for the Fourier transform of $\boldsymbol{u}(\boldsymbol{x})$. One can also demonstrate that the mean value of the Bloch amplitude is the Fourier amplitude of $\boldsymbol{u}(\boldsymbol{x})$ for the corresponding wave vector: $\langle\tilde{\boldsymbol{u}}(., \boldsymbol{k})\rangle_{\Omega_{x}}=\hat{\boldsymbol{u}}(\boldsymbol{k})$. Using the Bloch theorem to represent the solutions of periodical partial derivative equations implies that all derivatives are shifted by $k$ in the sense given by the used spatial operator.
Based on that theorem one can define the expansion functions $\boldsymbol{v}_{m}(\boldsymbol{x}, \boldsymbol{k})$, called the Bloch eigen modes, such that they can be used to represent the Bloch amplitudes of any solution of the corresponding partial derivative equation as

$$
\tilde{\boldsymbol{u}}(\boldsymbol{x}, \boldsymbol{k})=\sum_{m} \boldsymbol{u}_{m}(\boldsymbol{k}) \boldsymbol{v}_{m}(\boldsymbol{x}, \boldsymbol{k})
$$

and at the same time diagonalize the partial derivative equations. One notes that the expansion coefficients $\boldsymbol{u}_{m}(\boldsymbol{k})$ depend on the applied disturbance and also on the induced wave vector (see [29] for details).

\section{B. Application to Piezo-Elastodynamic}

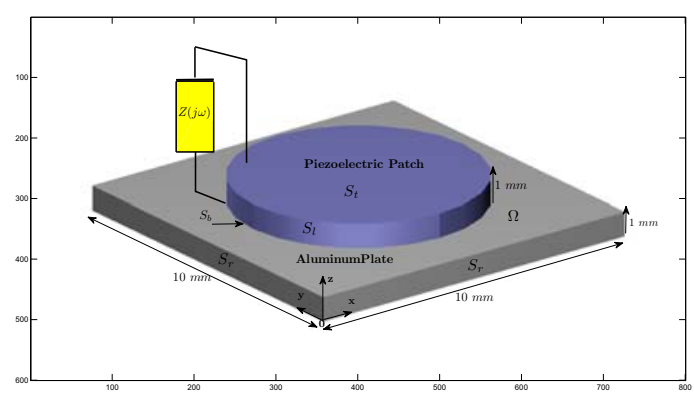

Fig. 1. Generic 3D piezocomposite periodic cell

Let us consider a piezo-elastodynamic problem made of infinite periodic distribution of unitary cell described in figure 1 . The harmonic homogeneous dynamical equilibrium of system is driven by the following partial derivative equation:

$$
\begin{cases}\rho \ddot{\boldsymbol{w}}(\boldsymbol{x})-\nabla \sigma(\boldsymbol{x})=0 & \forall \boldsymbol{x} \in \Omega_{x} \\ -\nabla D(\boldsymbol{x})=0 & \forall x \in \Omega_{x}\end{cases}
$$

where $\boldsymbol{w}(\boldsymbol{x}) \in \mathbb{R}^{3}\left(\Omega_{x}\right)$ is the displacement vector, $\sigma$ represents the Cauchy stress tensor, $\epsilon=\nabla_{\text {sym }} w=\frac{1}{2}\left(\nabla w^{T}(x)+\right.$ $\left.w(x) \nabla^{T}\right)$ the Green strain tensor, $D(\boldsymbol{x})$ the electric displacement. The linear constitutive material behavior relationships can be written as

$$
\begin{aligned}
\sigma & =C_{E}(\boldsymbol{x}) \epsilon-e^{T}(\boldsymbol{x}) \boldsymbol{E} \\
\boldsymbol{D} & =e(\boldsymbol{x}) \epsilon+\varepsilon_{S}(\boldsymbol{x}) \boldsymbol{E}
\end{aligned}
$$

where $\boldsymbol{E}=-\nabla V$ the electric field vector ( $V$ the voltage), $C_{E}$ the elasticity tensor at constant electrical field, $e^{T}$ the piezoelectric coupling tensor and $\varepsilon_{S}$ the dielectric permittivity at constant strain. We add to this set of equilibrium equations an output expression

$$
q^{o}=-\int_{S_{t}} \text { D.n. } d S
$$

allowing the introduction of the charge measurement on the piezoelectric's top electrode and hence the dual counterpart of the imposed electrical Dirichlet boundary condition for applying the shunt impedance operator.

The equations above are consistent for each kind of material 
to the extent that null piezoelectric and permittivity tensors can be used when passive materials are considered. All of these tensors also depend on the spatial location vector $\boldsymbol{x}$. The piezo-elastodynamic equilibrium can also be written as:

$\forall \boldsymbol{x} \in \Omega_{x}$

$$
\begin{aligned}
& \rho \omega^{2} \boldsymbol{w}(\boldsymbol{x})+\nabla C \nabla_{\text {sym }}(\boldsymbol{w}(\boldsymbol{x}))+\nabla e^{T}(\boldsymbol{x}) \nabla V(\boldsymbol{x})=0 \\
& -\nabla e(x) \nabla_{\text {sym }}(\boldsymbol{w}(\boldsymbol{x}))+\nabla \varepsilon_{S}(x) \nabla V(\boldsymbol{x})=0
\end{aligned}
$$

As the problem is $2 \mathrm{D}$ infinitely periodic, only electrostatic boundary conditions have to be considered on each cell:

$$
\begin{cases}V=0 & \forall x \in S_{b} \\ V=V^{o} & \forall x \in S_{t} \\ \text { D. } \boldsymbol{n}=0 & \forall x \in S_{l}\end{cases}
$$

where $S_{b}$ is the grounded bottom electrode of the piezoelectric layer, $S_{t}$ is the top electrode connected to the external shunt and $s_{l}$ the lateral electrode less boundary. The top electrode applied feedback voltage $V_{o}$ depends on the shunt characteristic and on the collected charges $q^{\circ}(7)$ and can be expressed in the Fourier space by:

$$
V^{o}(i \omega)=-Z(i \omega) q^{o}(i \omega)
$$

By considering a primitive cell of the periodic problem $\Omega_{x}$ and by using the Bloch theorem, we can compute the associated Bloch eigenmodes (3) and the dispersion functions by searching the eigen solutions of the homogeneous problem (8) and (9) as:

$$
\boldsymbol{u}(\boldsymbol{x})=\left[\begin{array}{c}
\boldsymbol{w}(\boldsymbol{x}) \\
V(\boldsymbol{x})
\end{array}\right]=\boldsymbol{u}_{n, \boldsymbol{k}}(\boldsymbol{x}) e^{i \boldsymbol{k} \cdot \boldsymbol{x}}
$$

with $\boldsymbol{u}_{n, \boldsymbol{k}}(\boldsymbol{x})=\left[\begin{array}{c}\boldsymbol{w}_{n, \boldsymbol{k}}(\boldsymbol{x}) \\ V_{n, \boldsymbol{k}}(\boldsymbol{x})\end{array}\right], \Omega_{x}$ periodic functions. By introducing expression (12) in the piezo-elastodynamic equations (8), (9), one can demonstrate that $\boldsymbol{w}_{n, \boldsymbol{k}}(\boldsymbol{x}), V_{n, \boldsymbol{k}}(\boldsymbol{x})$ and $\omega_{n}(\boldsymbol{k})$ are solutions of the generalized eigenvalues problem:

$$
\begin{aligned}
0 & =\rho \omega_{n}^{2}(\boldsymbol{k}) \boldsymbol{w}_{n, \boldsymbol{k}}(\boldsymbol{x})+\nabla C \nabla_{s y m}\left(\boldsymbol{w}_{n, \boldsymbol{k}}(\boldsymbol{x})\right) \\
& +i k\left\{\left(C \nabla_{s y m}\left(\boldsymbol{w}_{n, \boldsymbol{k}}(\boldsymbol{x})\right)\right) . \boldsymbol{\Phi}\right. \\
& \left.+\nabla\left(C \Xi_{n, \boldsymbol{k}}(\boldsymbol{x})\right)\right\}-k^{2}\left(C \Xi_{n, k}(\boldsymbol{x})\right) . \boldsymbol{\Phi} \\
& +\nabla e^{T} \nabla V_{n, \boldsymbol{k}}(\boldsymbol{x})+i k\left\{\left(\nabla e^{T} V_{n, \boldsymbol{k}}(\boldsymbol{x})\right) . \boldsymbol{\Phi}\right. \\
& \left.+\left(e^{T} \nabla V_{n, \boldsymbol{k}}(\boldsymbol{x})\right) . \boldsymbol{\Phi}\right\} \\
& -k^{2} V_{n, \boldsymbol{k}}(\boldsymbol{x})\left(e^{T} \boldsymbol{\Phi}\right) . \boldsymbol{\Phi} \forall \boldsymbol{x} \in \Omega_{x} \\
0 & -\nabla e \nabla_{s y m}\left(\boldsymbol{w}_{n, \boldsymbol{k}}(\boldsymbol{x})\right)-i k\left\{\nabla\left(e \Xi_{n, \boldsymbol{k}}(\boldsymbol{x})\right)\right. \\
+ & \left.\left(e \nabla_{s y m}\left(\boldsymbol{w}_{n, \boldsymbol{k}}(\boldsymbol{x})\right)\right) . \boldsymbol{\Phi}\right\} \\
+ & k^{2}\left(e \Xi_{n, \boldsymbol{k}}(\boldsymbol{x})\right) . \boldsymbol{\Phi} \\
+ & \nabla \varepsilon_{S} \nabla V_{n, \boldsymbol{k}}(\boldsymbol{x})+i k\left\{\left(\nabla \varepsilon_{S} V_{n, \boldsymbol{k}}(\boldsymbol{x})\right) . \boldsymbol{\Phi}\right. \\
+ & \left.\left(\varepsilon_{S} \nabla V_{n, \boldsymbol{k}}\right)(\boldsymbol{x}) . \boldsymbol{\Phi}\right\} \\
- & k^{2}\left(\varepsilon_{S} \boldsymbol{\Phi} V_{n, \boldsymbol{k}}(\boldsymbol{x})\right) . \boldsymbol{\Phi} \forall \boldsymbol{x} \in \Omega_{x}
\end{aligned}
$$

with the associated boundary conditions:

$$
\begin{cases}\boldsymbol{w}_{n, \boldsymbol{k}}(\boldsymbol{x}-R \cdot \boldsymbol{m})=\boldsymbol{w}_{n, \boldsymbol{k}}(\boldsymbol{x}) & \forall \boldsymbol{x} \in S_{r} \boldsymbol{m} \in \mathbb{Z}^{2} \\ V_{n, \boldsymbol{k}}(\boldsymbol{x})=0 & \forall \boldsymbol{x} \in S_{b} \\ V_{n, \boldsymbol{k}}(\boldsymbol{x}) V=-Z(i \omega) q_{n, \boldsymbol{k}}^{o} & \forall \boldsymbol{x} \in S_{t} \\ \boldsymbol{D} \boldsymbol{n}=0 & \forall \boldsymbol{x} \in S_{l}\end{cases}
$$

where $\boldsymbol{k}=k\left[\begin{array}{c}\cos (\phi) \\ \sin (\phi) \\ 0\end{array}\right]=k \boldsymbol{\Phi}$ where $\phi$ represents the direction angle into the reciprocal 2D lattice domain and $\Xi_{n, \boldsymbol{k}}(\boldsymbol{x})=$ $\frac{1}{2}\left(\boldsymbol{w}_{n, \boldsymbol{k}}(\boldsymbol{x}) \cdot \Phi^{T}+\Phi \cdot \boldsymbol{w}_{n, \boldsymbol{k}}^{T}(\boldsymbol{x})\right)$ the symmetric dyadic tensor or the dyadic product of the displacement $\boldsymbol{w}_{n, \boldsymbol{k}}(\boldsymbol{x})$ and direction vector $\Phi . S_{r}$ are the interface of the cells continuum, and $R$ the matrix grouping the two lattice's basis vectors (in 2D in the considered problem). In the electrical boundary conditions, $q_{n, \boldsymbol{k}}^{o}$ is given by:

$$
\begin{aligned}
q_{n, \boldsymbol{k}}^{o} & =\int_{S_{t}}\left[-e\left(\nabla_{s y m}\left(\boldsymbol{w}_{n, \boldsymbol{k}}(\boldsymbol{x})\right)+i k \nabla e \Xi_{n, \boldsymbol{k}}(\boldsymbol{x})\right)\right. \\
& +\varepsilon_{S}\left(\nabla V_{n, \boldsymbol{k}}(\boldsymbol{x})+i k V_{n, \boldsymbol{k}}(\boldsymbol{x}) \boldsymbol{\Phi}\right] . \boldsymbol{n} d S
\end{aligned}
$$

where $\boldsymbol{n}$ is the outpointing unitary normal vector.

The proposed formulation is also based on the computation of the Floquet vectors (13), (14), instead of computing the Floquet propagators commonly used for elastodynamic applications. Our approach allows to obtain the full 2D waves dispersions functions and to clearly introduce damping and electrical impedance into the piezo-elastodynamic operator. The adopted methodology allows the computation of the complete complex map of the dispersion curves including evanescent waves and allowing the introduction of damping and shunt operator if any.

\section{Weak formulation and computation of waves dispersion functions in periodical piezo-composite lattice}

Let us consider the partial derivative equations (13), (14) on a unit cell $\Omega$. It stands for a generalized eigenvalue problem leading to the computation of the dispersion functions $\omega_{n}(\boldsymbol{k})$ and the corresponding Floquet eigenvectors $\boldsymbol{u}_{n, \boldsymbol{k}}(\boldsymbol{x})$. For computing the 2D dispersions curves, we need to introduce a suitable weak formulation.

$$
\begin{aligned}
& \text { If } \quad \boldsymbol{u}_{n, \boldsymbol{k}}(\boldsymbol{x}) \quad \text { is a solution of } \\
& \text { tions }(13), \quad(14), \quad \text { also } \forall \tilde{\boldsymbol{w}}_{n, \boldsymbol{k}}(\boldsymbol{x}) \\
& \left\{H_{1}\left(\Omega, \mathbb{C}^{3}\right) / \tilde{\boldsymbol{w}}_{n, \boldsymbol{k}}(\boldsymbol{x}-R \boldsymbol{m})=\tilde{\boldsymbol{w}}_{n, \boldsymbol{k}}(\boldsymbol{x}) \forall \boldsymbol{x} \in S_{r}\right\} \\
& \text { and } \tilde{V}_{n, \boldsymbol{k}}(\boldsymbol{x}) \in\left\{H_{1}(\Omega, \mathbb{C}) / \tilde{V}_{n, k}(\boldsymbol{x})=0 \forall \boldsymbol{x} \in S_{b}\right. \text { and }
\end{aligned}
$$




$$
\begin{aligned}
& \left.\tilde{V}_{n, k}(\boldsymbol{x})=V^{o} \forall \boldsymbol{x} \in S_{t}\right\} \text { we have: } \\
& 0=\int_{\Omega} \rho \omega_{n}^{2}(\boldsymbol{k}) \tilde{\boldsymbol{w}}_{n, \boldsymbol{k}}(\boldsymbol{x}) \boldsymbol{w}_{n, \boldsymbol{k}}(\boldsymbol{x}) \\
& -\left(\tilde{\varepsilon}_{n, \boldsymbol{k}}(\boldsymbol{x})-i k \tilde{\Xi}_{n, \boldsymbol{k}}(\boldsymbol{x})\right) C\left(\varepsilon_{n, \boldsymbol{k}}(\boldsymbol{x})+i k \Xi_{n, \boldsymbol{k}}(\boldsymbol{x})\right) \\
& +\left(\tilde{\varepsilon}_{n, \boldsymbol{k}}(\boldsymbol{x})-i k \tilde{\Xi}_{n, \boldsymbol{k}}(\boldsymbol{x})\right) e^{T}\left(\nabla V_{n, \boldsymbol{k}}(\boldsymbol{x})+i k V_{n, \boldsymbol{k}}(\boldsymbol{x}) \boldsymbol{\Phi}\right) \\
& -\left(\nabla \tilde{V}_{n, \boldsymbol{k}}(\boldsymbol{x})-i k \tilde{V}_{n, \boldsymbol{k}}(\boldsymbol{x}) \boldsymbol{\Phi}\right) e\left(\varepsilon_{n, \boldsymbol{k}}(\boldsymbol{x})+i k \Xi_{n, \boldsymbol{k}}(\boldsymbol{x})\right) \\
& +\left(\nabla \tilde{V}_{n, \boldsymbol{k}}(\boldsymbol{x})-i k \tilde{V}_{n, \boldsymbol{k}}(\boldsymbol{x}) \boldsymbol{\Phi}\right) \varepsilon_{S}\left(\nabla V_{n, \boldsymbol{k}}(\boldsymbol{x})+i k V_{n, \boldsymbol{k}}(\boldsymbol{x}) \boldsymbol{\Phi}\right) d \Omega \\
& -\frac{\tilde{V}_{n, \boldsymbol{k}}^{t} V_{n, \boldsymbol{k}}^{t}}{Z(i \omega)}
\end{aligned}
$$

This weak formulation is simply obtained by integrating equation (13), (14) projected onto any test function $\tilde{\boldsymbol{u}}_{n, \boldsymbol{k}}(\boldsymbol{x})$. The boundary integral vanishes as the test functions are chosen so that $\tilde{\boldsymbol{w}}_{n, \boldsymbol{k}}(\boldsymbol{x}-R \boldsymbol{m})=\tilde{\boldsymbol{w}}_{n, \boldsymbol{k}}(\boldsymbol{x})$ on $S_{r}$. For a polyhedron cell, each boundary is generally a polyhedral plane sub-domain that can be associated with its parallel opposite one. The symmetry conditions $\tilde{\boldsymbol{w}}_{n, \boldsymbol{k}}(\boldsymbol{x}-R \boldsymbol{m})=\tilde{\boldsymbol{w}}_{n, \boldsymbol{k}}(\boldsymbol{x})$ explicitly link these associated surfaces.

\section{Numerical Computation of the Bloch's waves}

The numerical implementation is obtained by using a standard finite elements method to discretize the weak formulation (16). The assembled matrix equation is given by:

$$
\begin{aligned}
0 & =\left(K \left(Z\left(i \omega_{n}(\lambda, \phi)\right)+\lambda L\left(\phi, Z\left(i \omega_{n}(\lambda, \phi)\right)\right)\right.\right. \\
& \left.-\lambda^{2} H\left(\phi, Z\left(i \omega_{n}(\lambda, \phi)\right)\right)-\omega_{n}^{2}(\lambda, \phi) M\right) \boldsymbol{u}_{n, k}(\phi),
\end{aligned}
$$

where $\lambda=i k, M$ and $K\left(Z\left(i \omega_{n}(\lambda, \phi)\right)\right)$ are respectively the standard symmetric semi-definite mass and stiffness matrices (the mass matrix is semi definite because elastostatic equation are condensed into the equation), $L\left(\phi, Z\left(i \omega_{n}(\lambda, \phi)\right)\right)$ is a skew-symmetric matrix and $H\left(\phi, Z\left(i \omega_{n}(\lambda, \phi)\right)\right)$ is a symmetric semi-definite positive matrix

When $k$ and $\phi$ are fixed and $Z$ does not depend on $\omega$ the system (17) is a linear eigen value problem allowing us to compute the dispersion functions $\omega_{n}^{2}(\boldsymbol{k}, \phi)$ and the associated Bloch eigenvector $\boldsymbol{u}_{n, k}(\phi)$.

This approach has been widely used for developing homogenization techniques and spectral asymptotic analyses like in the work of [30]. It can also be applied for computing wave's dispersion even if Floquet propagators is preferred for 1D or quasi 1D computation, as indicated in [31], [32], [33]. Nevertheless these approaches have been only developed for undamped or lightly damped mechanical systems. In these cases, most of the previously published works present techniques based on the mesh of a real $k$-space (i.e $k$ or $\lambda$ and $\phi$ ) following the boundary of the first Brillouin zone for obtaining the corresponding frequency dispersion and the associated Floquet vectors. For undamped system, only propagative or evanescent waves exist corresponding to a family of eigen solutions purely real or imaginary. Discrimination between each class of waves is easy. If a highly damped system $(K, L, H$ are complex frequency dependent) and a frequency dependent electrical shunt impedance are considered, the obtained eigenvalue problem is not quadratic and a complex specific numerical methodology has to be implemented. Furthermore, evanescent part of propagating waves appear as the imaginary part of eigenfrequencies. It then becomes very difficult to distinguish the propagative and evanescent waves as all solution appear complex.

Another much more suitable possibility for computing dis$\Omega$ persion in damped systems, dedicated for time/space deconvolution and for computation of diffusion properties as defined by [4], [33], is to consider the following generalized eigen value problem:

$$
\begin{aligned}
0 & =\left(K \left(Z(\omega)-\omega^{2} M+\lambda_{n}(\omega, \phi) L(\phi, Z(\omega))\right.\right. \\
& \left.-\lambda_{n}^{2}(\omega, \phi) H(\phi, Z(\omega))\right) \boldsymbol{u}_{n}(\omega, \phi) .
\end{aligned}
$$

In this problem, the pulsation $\omega$ is a real parameter corresponding to the harmonic frequency. Wave's numbers and Floquet vectors are then computed. An inverse Fourier transformation in the $k$-space domain can lead us to evaluate the physical wave's displacements and energy diffusion operator when the periodic distribution is connected to another system as in [4]. Another temporal inverse Fourier transformation can furnish a way to access spatio-temporal response for non-homogeneous initial conditions. As $L$ is skew-symmetric, the obtained eigen values are quadruple $(\lambda, \bar{\lambda},-\lambda,-\bar{\lambda})$ collapsing into real or imaginary pairs (or a single zero) when all matrices are real (i.e. for an undamped system). In this case a real pair of eigen values correspond to evanescent modes oriented in two opposite directions on the $k$-space and imaginary values to two traveling waves propagating in opposite direction.

As previously mentioned, the real part of $\boldsymbol{k}=k \boldsymbol{\Phi}$ vector is restricted to stand inside the first Brillouin zone. In the quadratic eigen value problem (18) nothing restricts computation to only find eigen values satisfying this condition. For direction vector $\boldsymbol{\Phi}$ orthogonal to the lattice facelets (i.e. for $\Phi_{p 1}=[1,0]^{T}$ and $\Phi_{p 2}=[0,1]^{T}$ in bi-dimensional rectangular cell), the periodical conditions expressed for one dimensional wave guide are still valid: if $\lambda_{j}\left(\omega, \boldsymbol{\Phi}_{p}\right)$ is an eigen value associated to $\boldsymbol{w}_{j}\left(\omega, \boldsymbol{\Phi}_{p}\right)$ then $\forall \boldsymbol{m} \in \mathbb{Z}^{3}, \lambda+i . \Phi_{p}^{T}(G . \boldsymbol{m})$ is also an eigen value associated to $\boldsymbol{w}_{j}\left(\omega, \boldsymbol{\Phi}_{p}\right) e^{-i \cdot \boldsymbol{\Phi}_{p}^{T}(\text { G.m }) x}$. Thus, for undamped systems, all obtained eigenvalues are periodically distributed in the $\boldsymbol{k}$-space along its principal directions.

\section{E. Computation of the evanescence and damped power flow criteria}

One aim of this paper is to provide a numerical methodology for optimizing the piezoelectric shunt impedance $Z(\omega)$ for controlling energy flow into the periodically distributed piezocomposite structure. For doing this, we need to define suitable criteria.

The first used criterion is based on the computation of the waves group velocities. Indeed, they indicate how energy is transported into the considered system and allow to distinguish the 'propagative' and 'evanescent' waves. If one Bloch eigen 
solution (i.e $\boldsymbol{u}_{n}(\omega, \phi), k_{n}(\omega)$ ) is considered, the associated group velocity vector [34] is given by:

$$
\boldsymbol{C}_{g_{n}}(\omega, \phi)=\nabla_{\boldsymbol{k}} \omega=\frac{\langle\langle\boldsymbol{S}\rangle\rangle}{\left\langle\left\langle e_{t o t}\right\rangle\right\rangle}=\frac{\langle\boldsymbol{I}\rangle}{\left\langle E_{t o t}\right\rangle}
$$

where $\langle\langle:\rangle\rangle$ is the spatial and time average respectivelly on one cell and one period, $S$ is the density of energy flux defined in [34], $\boldsymbol{I}$ the mean intensity and $e_{t o t}, E_{t o t}$ the total piezomechanical energy and its time average on a period (see [34] for details). In this problem, we only consider mechanical energy transportation as the electrostatic coupling is decentralized and can be condensed as a mechanical interface as proved in [35] and generally computed in [36]. So we also compute the intensity vector $\boldsymbol{I}$ by:

$$
\left\langle\boldsymbol{I}_{n}\right\rangle(\omega, \phi)=-\frac{\omega}{2 V_{o l}} \operatorname{Re}\left(\int_{\Omega_{x}} C\left(\varepsilon_{n}(\boldsymbol{x})+i k \Xi_{n}(\boldsymbol{x})\right) \cdot\left(\boldsymbol{w}_{n}^{*}(\boldsymbol{x})\right)\right) d \Omega
$$

where.$^{*}$ is the complex conjugate and $V_{o l}$ the domain volume. As the spatio-temporal average of the system Lagragian is null (see [34]), the total energy average is approximated by only computing the kinetic energy average:

$$
\left\langle E_{t o t}\right\rangle(\omega, \phi)=\frac{1}{2 V_{o l}} \operatorname{Re}\left(\int_{\Omega_{x}} \rho \omega^{2} \boldsymbol{w}_{n}(\boldsymbol{x}) \cdot \boldsymbol{w}_{n}^{*}(\boldsymbol{x} i) d \Omega\right)
$$

The group velocity vectors $C_{g_{n}}(\omega, \phi)$ is computed for all wave numbers at each frequency. In order to focus our analysis on only flexural modes ( $S$ and $S H$ ones) we introduce an indicator allowing to select them by computing the ratio of kinetic energy average on out of plane displacement as:

$$
\operatorname{Ind}(n, \omega, \phi)=\frac{\frac{1}{2 V_{o l}}\left(\int_{\Omega_{x}} \rho \omega^{2} w z_{n}(\boldsymbol{x}) w z_{n}^{*}(\boldsymbol{x}) d \Omega\right)}{\left\langle E_{\text {tot }}\right\rangle}
$$

with $w z_{n}(\boldsymbol{x}, \omega, \phi)$ being the $(O z)$ component of vector $\boldsymbol{w}_{n}(\boldsymbol{x}, \omega, \phi)$. Optimization of the shunt impedance $Z(i \omega)$ is based on the minimization of the maximum group velocity collinear to the wave number vector (19) for waves having a ratio of transported flexural kinetic energy (22) greater than 0.8 . The used criterion can also be written as:

$$
\operatorname{Crit}_{1}(Z(i \omega), \phi)=\max _{n / \operatorname{Ind}(n, \omega, \phi)>0.8}\left(\boldsymbol{C}_{g_{n}}(\omega, \phi) . \boldsymbol{\Phi}\right)
$$

The second used criterion is based on the maximization of the damped electric power directly express as the active electrical power $P_{\text {elec }}(n, \omega, \phi)=\frac{1}{2} \operatorname{real}\left(i \omega Z(i \omega) q_{n, \boldsymbol{k}}^{o} q_{n, \boldsymbol{k}}^{o *}\right)$. If one wants to increase damping effect inside the smart metamaterial, this term need to be sufficiently large. In the second case, the used criteria is also

$$
\operatorname{Crit}_{2}(Z(i \omega), \phi)=\max _{n / \operatorname{Ind}(n, \omega, \phi)>0.8} \frac{1}{P_{\text {elec }}(n, \omega, \phi)}
$$

\section{F. Computation of the sound radiation efficiency}

The sound radiation efficiency of a plate depends on the coupling of sound waves in the air and flexural waves (vibration) in the plate. Optimum efficiency (maximum energy transfer from vibration to sound or vice versa) is achieved when the plate vibrates such that the wavelength of flexural

\begin{tabular}{||l|l|l||}
\hline \multicolumn{3}{|c||}{ Piezoelectric Material } \\
\hline \hline Symbol & Value & Property \\
\hline$s_{11}^{E}=s_{22}^{E}=s_{33}^{E}$ & $11.6 e^{-12} P a^{-1}$ & compliance matrix \\
\hline$s_{12}^{E}=s_{13}^{E}=s_{23}^{E}$ & $-3.33 e^{-12} \mathrm{~Pa}^{-1}$ & compliance matrix \\
\hline$s_{44}^{E}=s_{55}^{E}=s_{66}^{E}$ & $45.0 e^{-11} P a^{-1}$ & compliance matrix \\
\hline$\eta$ & $0.1 \%$ & Hysteretic Damping ratio \\
\hline$d_{31}=d_{32}$ & $-6 e^{-11} \mathrm{C} / \mathrm{N}$ & piezoelectric matrix \\
\hline$d_{33}$ & $15.2 e^{-11} \mathrm{C} / \mathrm{N}$ & piezoelectric matrix \\
\hline$d_{24}=d_{15}$ & $730 e^{-12} \mathrm{C} / \mathrm{N}$ & piezoelectric matrix \\
\hline$\rho$ & $7600 \mathrm{~kg} / \mathrm{m}^{3}$ & Density \\
\hline$\varepsilon_{11}^{T}=\varepsilon_{22}^{T}$ & $504.1 \varepsilon_{o} \mathrm{C} / \mathrm{V} / \mathrm{m}$ & Dielectric Permittivity \\
$\varepsilon_{33}^{T}$ & $270 \varepsilon_{o} C / \mathrm{V} / \mathrm{m}$ & Dielectric Permittivity \\
\hline
\end{tabular}

TABLE I

Piezoelectric patch characteristics

waves in the plate is equal to the wavelength of acoustic waves in the air (alternatively the flexural wave velocity in the plate is equal to the velocity of acoustic waves in the air). This is more commonly known as the coincidence or critical frequency of radiation. In fact, that phenomenon is based on the computation of the $k z_{n}$ value of the induced acoustic wave number. If one considers an infinite plate in which an harmonic plane wave is propagating with a wave number $k x_{n}$ at pulsation $\omega$, as the acoustic pressure radiated from this infinite system is a solution of the Helmoltz equation and is coupled by considering interface continuous normal velocity, one can obtain expression of $k z_{n}$ by:

$$
k z_{n}=\sqrt{\left(\frac{\omega}{c}\right)^{2}-k x_{n}^{2}}
$$

where $c$ is the sound velocity in the considered semi-infinite acoustic domain.

For analyzing sound radiation effect of our smart metamaterial, acoustic wave numbers $k z_{n}$ are post-processed from the obtained structural waves given by our previously described computation. As the obtained $k x_{n}$ are complex and include all modes, we will focus our analysis to flexural modes by using indicator $\operatorname{Ind}(n, \omega, \phi)>0.8$ and consider the complex expression of the out of plane radiation by using equation (25) with a complex $k z_{n}$.

\section{OPTIMIZATION OF THE FLEXURAL ENERGY FLOW INSIDE THE SHUNTED PERIODIC PIEZO-COMPOSITE}

The considered piezo-composite cell is presented in figure 1. The supporting plate material is standard aluminum with 0.1 $\%$ of hysteretic damping ratio and the piezoelectric material characteristics are given in table I.

The used numerical optimizations of the criteria are based on a multidimensional unconstrained nonlinear minimization (Nelder-Mead).

\section{A. Optimization of the waves goupes velocities by using Crit $_{1}$}

In a first test, we optimize the waves groups velocities by using Crit $_{1}$ given in equation (23). We present in figure 2 the obtained real and imaginary parts of the wave number $k x_{n}(i \omega)$ of plane waves propagating into the smart plate 

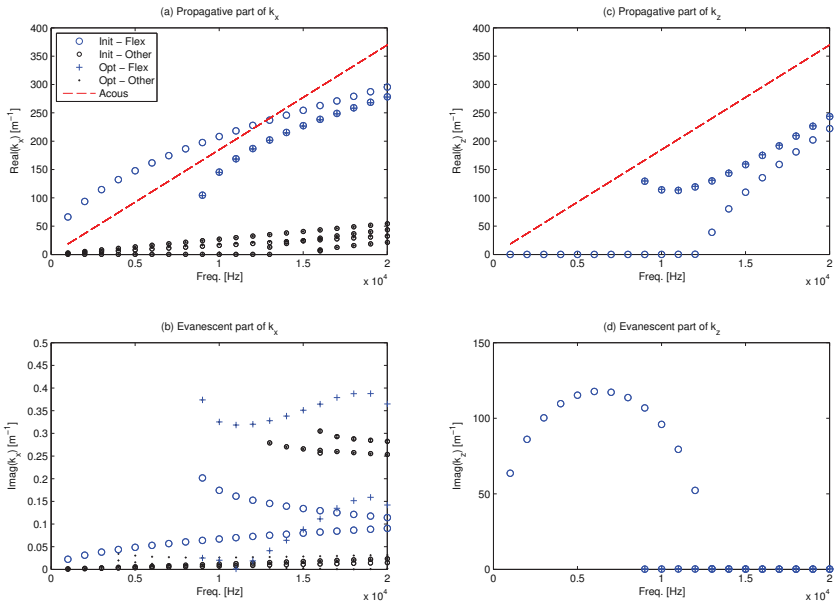

Fig. 2. Real (a) and imaginary (b) parts of the wave number $k x_{n}(i \omega)$ of plane waves propagating into the smart plate along $(O x)$ axis and the corresponding real (a) and imaginary (b) parts of the acoustic out plane wave number $k z_{n}$.
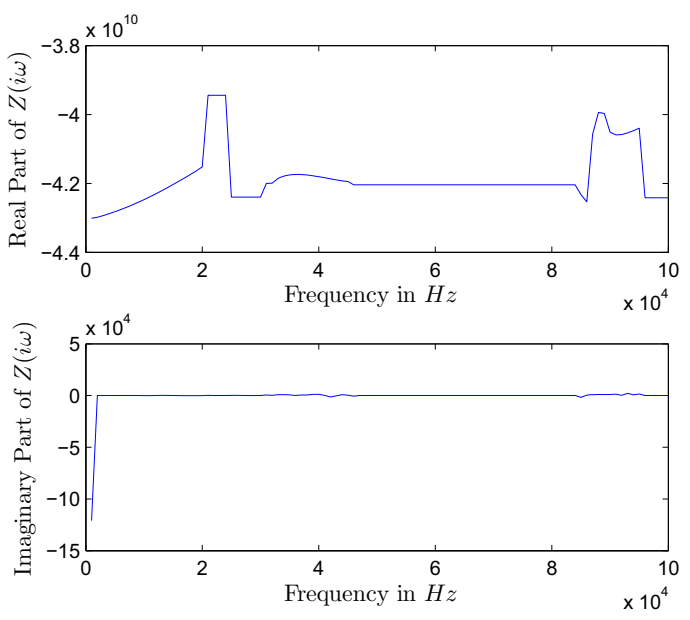

Fig. 3. Real and imaginary parts of the optimal impedance

along $(O x)$ axis and the corresponding real and imaginary parts of the acoustic out of plane wave number $k z_{n}$. The red dashed lines indicate the acoustic wave number $\frac{\omega}{c}$. The circles mark the dispersions curves for $Z=0$ and the blue crosses the obtained optimal dispersion. The corresponding real and imaginary parts of the optimal impedance are plotted in figure 3. For a sake of clarity, all non propagative waves having a group velocity inferior to $30 \mathrm{~m} . \mathrm{s}^{-1}$ have been removed from the plotted results.

We immediately observe that the optimization of the shunt impedance leads to greatly modify the group velocity of the $A_{o}$ mode and to create an evanescent mode. We notice an increase of the imaginary parts of the wave number of $A_{1}$ mode indicating an increase of the spatial decay rates even if this mode remains propagative. The acoustic radiation is greatly modified by the cancellation of $A_{o}$ mode as shown
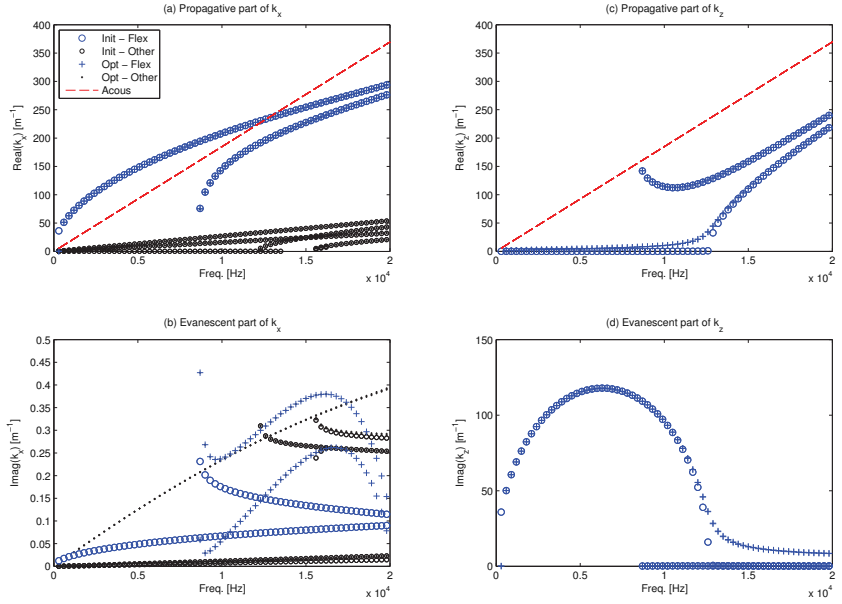

Fig. 4. Real (a) and imaginary (b) parts of the wave number $k x_{n}(i \omega)$ of plane waves propagating into the smart plate along $(O x)$ axis and the corresponding real (a) and imaginary (b) parts of the acoustic out plane wave number $k z_{n}$

in figure 2.c. The imaginary part of $k z_{n}$ stay unchanged at very low values for radiations induced by $A_{1}$. The optimal impedance values are almost real, and correspond to those obtained if a constant negative capacitance is used. The corresponding average value is $-150.05 p C . V^{-1}$. Some imaginary parts of the optimal impedance are negative which indicate that the optimization leads to provide energy to the system for controlling mechanical damping effect introduced with hysteretic damping ratios into the model, and, also, obtain a fully conservative system.

B. Optimization of damped power flow inside the electric shunts by using $\mathrm{Crit}_{2}$

In this second test, we optimize the damped power flow inside the electric shunts by using $\mathrm{Crit}_{2}$ given in equation (24). We present in figure 4 the obtained real and imaginary parts of the wave number $k x_{n}(i \omega)$ of plane waves propagating into the smart plate along $(O x)$ axis and the corresponding real and imaginary parts of the acoustic out of plane wave number $k z_{n}$. The red dashed lines mark the acoustic wave number $\frac{\omega}{c}$. The circles mark the dispersions curves for $Z=0$ and the blue crosses the obtained optimal dispersion. The corresponding real and imaginary parts of the optimal impedance are plotted in figure 3. For a sake of clarity, all non propagative waves having a group velocity inferior to $30 \mathrm{~m} . \mathrm{s}^{-1}$ have been removed from the plotted results.

We immediately observe that the optimization of the shunt impedance leads to greatly modify the imaginary parts of the flexural wave number of $A_{o}$ and $A_{1}$ modes and to create damped propagation while the real parts remain unchanged. The acoustic radiation is greatly modified in improving the spatial decay rates of the out of plane wave number coupled with $A_{o}$ mode as shown in figure $4 . \mathrm{d}$ after $12 \mathrm{kHz}$. The real parts of $k z_{n}$ stay unchanged. The optimal impedance values are complex with a positive resistance term that allows energy 

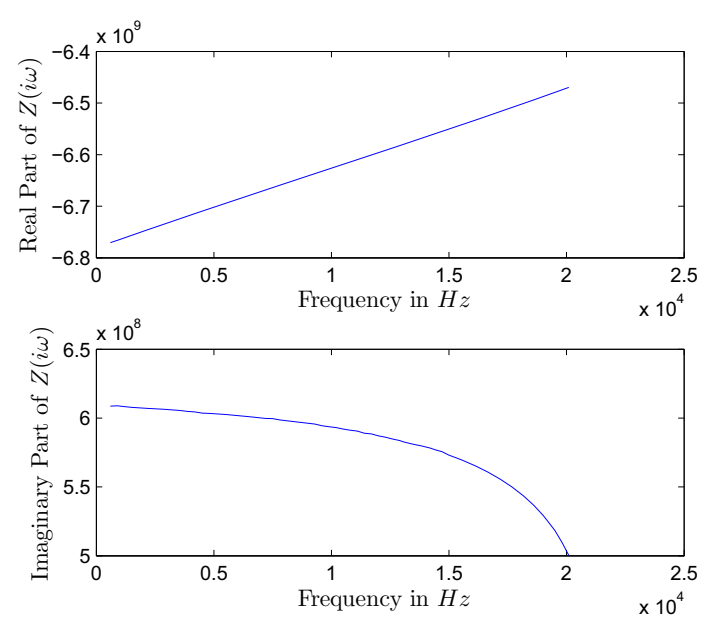

Fig. 5. Real and imaginary parts of the optimal impedance

dissipation inside the electric shunt circuit. The real parts correspond to those obtained if a constant negative capacitance is used. The corresponding average value is also -151.04 $p C . V^{-1}$ slightly different from the previously considered case.

\section{CONCLUSIONS}

This paper presents a numerical procedure able to compute the damped wave's dispersion functions in the whole first Brillouin domain of multi dimensionnal piezo-elastodynamical wave guides. The method was applied for determining the optimal impedance allowing to minimize the group velocities of the flexural waves. Based on this approach, some numerical tests on a finite dimension system incorporating a semidistributed set of shunted piezo-composite cells have been performed. We underline a strong influence of the designed shunt circuits in the dynamical response of the system and the coupled noise radiation. Even if the link between the obtained wave properties are not clearly established, we also demonstrated that our developed numerical procedures can be used for optimizing the energy diffusion operator of such adaptive mechanical interface. To do so, additional work has to be done for optimizing the complete interface scattering and for controlling the evanescent waves playing an important role in the dynamical response of a finite system incorporating such semi-distributed interface. Another part of this future developments should deal with the complete vibroacoustic optimization incorporating a fully fluid-structure coupling effect. The proposed methodology can also be used for studying particular dissipation phenomenon such as those induced by complex shunted piezoelectric patches as proposed by [6] and [37], or even foams or complex polymers behaviors. The proposed method furnishes an efficient tool for future optimization of distributed smart cells as proposed in the case of $1 \mathrm{D}$ wave guide by [4].

\section{ACKNOWLEDGMENT}

This work was carried out with a grant of French agency ANR number NT09-617542. We gratefully acknowledge the French ANR and CNRS for supporting this program.

\section{REFERENCES}

[1] A. Preumont, Vibration control of structures : An introduction. Kluwer, 1997.

[2] S. P.A. Nelson, Active Control of Sound. London, San Diego: Pub. Academic Press, 1992.

[3] H. Banks and Y. W. R.C. Smith, Smart material structures Modeling Estimation and Control. Masson and Wiley, 1996.

[4] M. Collet, K. Cunefare, and N. Ichchou, "Wave Motion Optimization in Periodically Distributed Shunted Piezocomposite Beam Structures," Journal of Int Mat Syst and Struct, vol. 20, no. 7, pp. 787-808, 2009.

[5] O. Thorp, M. Ruzzene, and A. Baz, "Attenuation and localization of wave propagation in rods with periodic shunted piezoelectric patches," Proceedings of SPIE - The International Society for Optical Engineering Smart Structures and Materials, vol. 4331, pp. 218-238, 2001.

[6] B. Beck, K. Cunefare, and M. Ruzzene, "Broadband vibration suppression assessment of negative impedance shunts," in Proceedings of SMASIS08, vol. 6928. ASME, 2008.

[7] B. Beck, K. A. Cunefare, M. Ruzzene, and M. Collet, "Experimental analysis of a cantilever beam with a shunted piezoelectric periodic array," in ASME-SMASIS. Philadelphia: ASME, Sept 28 Oct 12010.

[8] I. Bartoli, A. Marzani, F. L. di Scalea, and E. Viola, "Modeling wave propagation in damped waveguides of arbitrary cross-section," Journal of Sound and Vibration, vol. 295, no. 3-5, pp. 685-707, AUG 222006.

[9] M. Foda, A. Almajed, and M. ElMadany, "Vibration suppression of composite laminated beams using distributed piezoelectric patches," Smart Materials and Structures, vol. 19, no. 11, p. 115018, 2010 [Online]. Available: http://stacks.iop.org/0964-1726/19/i=11/a=115018

[10] M. S. I. S. Dawood, L. Iannucci, and E. S. Greenhalgh, "Threedimensional static shape control analysis of composite plates using distributed piezoelectric actuators," Smart Materials and Structures, vol. 17 , no. 2, p. 025002, 2008. [Online]. Available: http://stacks.iop.org/0964-1726/17/i=2/a=025002

[11] J. Jiang and D. Li, "Decentralized robust vibration control of smart structures with parameter uncertainties," Journal of Intelligent Material Systems and Structures, 2010. [Online]. Available: http://jim.sagepub.com/content/early/2011/01/07/1045389X10391496.abstract

[12] B. Y. Ren, L. Wang, H. S. Tzou, and H. H. Yue, "Finite difference based vibration simulation analysis of a segmented distributed piezoelectric structronic plate system," Smart Materials and Structures, vol. 19, no. 8 , p. 085024, 2010. [Online]. Available: http://stacks.iop.org/0964$1726 / 19 / \mathrm{i}=8 / \mathrm{a}=085024$

[13] D. Mead, "A general theory of harmonic wave propagation in linear periodic systems with multiple coupling," J. Sound Vibrat., vol. 27, no. 2, pp. 429-438, 1996.

[14] R. Ohayon, "Vibration analysis of periodic structures and brillouin zones computation. finite element applications to stiffened cylinders," in Proc. 16th IUTAM Congress, Lungby, Danmark, University of Denmark, Lungby, 1984

[15] B. Mace and E. Manconi, "Modelling wave propagation in twodimensional structures using finite element analysis," Journal of Sound and Vibrations, vol. 318, pp. 884-902, 2008.

[16] S. Gonella and M. Ruzzene, "Analysis of in-plane wave propagation in hexagonal and re-entrant lattices," J. Sound Vibrat., vol. 312, pp. 125139, 2008.

[17] S. Akrout, "Comportement dynamique dterministe et large bande des structures guides," Ph.D. dissertation, cole Centrale Lyon, 2005.

[18] E. Manconi, "The wave finite element method for 2-dimensional structures," Ph.D. dissertation, University of Parma, 2008.

[19] J. Berthaut, M. Collet, and M. Ichchou, "Multi-mode wave propagation in ribbed plates : Part I k-space characteristics," International Journal of Solids and Structures, vol. 45, no. 5, pp. 1179-1195, 2008.

[20] R. Ohayon and F. Jouaillec, "Fluid structure vibrations analysis of infinite periodic stiffened cylinders," Proc. ASME- PVP New Orleans, vol. 98-7, pp. 185-188, 1985.

[21] R. Ohayon, "Fluid structure interaction analysis for cyclically symmetric bounded systems," Proc. ASME- PVP New Orleans, vol. 98-7, pp. 179184, 1985. 
[22] M. Collet, M. Ouisse, M. Ruzzene, and M. Ichchou, "A FloquetBloch decomposition of the elastodynamical equations: application to bi-dimensional wave's dispersion computation of damped mechanical system." International Journal of Solids and Structures, vol. In press, 2011.

[23] M. Collet, M. Ouisse, M. Ichchou, and M. Ruzzene, "Semi-active optimization of $2 \mathrm{~d}$ waves dispersion into mechanical systems by the mean of periodically distributed shunted piezoelectric patches: a new class of adaptive metamaterials,", in SPIE Smart Structures/NDE, SanDiego (CA), 2011

[24] G. Floquet, "Sur les équations différentielles linéaires á coefficients périodiques," Annales de l'Ecole Normale Supérieure, vol. 12, pp. 47$88,1883$.

[25] F. Bloch, "Über die Quantenmechanik der Electron in Kristallgittern," Zeitschrift für Physik, vol. 52, pp. 550-600, 1928.

[26] M. Collet, M. Ouisse, M. Ichchou, and M. Ruzzene, "Numerical tools for semi-active optimization of $2 \mathrm{~d}$ waves dispersion into mechanical system," in ASME-SMASIS. Philadelphia: ASME, Sept 28 Oct 12010.

[27] J. Joannopoulos, R. Meade, and J. Winn, Photonic Crystals: Molding the Flow of Light. Princeton University Press, 1995.

[28] C. Kittel, Introduction to Solid State Physics. John Wiley and Sons, New York, 1986.

[29] A. Bensoussan, J. Lions, and G. Pananicolaou, Asymptotic Analysis for Periodic Structures. North Holland, 1978.

[30] G. Allaire and C. Congas, "Bloch waves homogenization and spectral asymptotic analysis," Journal de Mathmatiques Pures et Appliques, vol. 77, pp. 153-208, 1998.

[31] M. N. Ichchou, S. Akrout, and J. Mencik, "Guided waves group and energy velocities via finite elements," Journal of Sound and Vibration, vol. 305, no. 4-5, pp. 931-944, 2007.

[32] L. Houillon, M. Ichchou, and L. Jezequel, "Wave motion in thin-walled structures," Journal of Sound and Vibration, vol. 281, no. 3-5, pp. 483507, 2005.

[33] J. Mencik and M. Ichchou, "Multi-mode propagation and diffusion in structures through finite elements," European Journal of Mechanics ASolids, vol. 24, no. 5, pp. 877-898, 2005.

[34] W. Maysenhölder, Körperschall-energie Grundlagen zur Berechnung von Energiedichten und Intensitäten. Wissenschaftliche Verlagsgesellschaft, Stuttgart, 1994.

[35] N. W. Hagood and A. H. von Flotow, "Damping of structural vibrations with piezoelectric materials and passive electrical networks," Journal of Sound and Vibration, vol. 146(2), pp. 243-268, 1991.

[36] M. Collet and K. Cunefare, "Modal Synthesis and Dynamical Condensation Methods for Accurate Piezoelectric Systems Impedance Computation," Journal of Int Mat Syst and Struct, vol. 19, no. 11, pp. 1251-1271, 2008.

[37] F. Casadei, M. Ruzzene, B. Beck, and K. Cunefare, "Vibration control of plates featuring periodic arrays of hybrid shunted piezoelectric patches," in Proceedings of SPIE - Smart Structures and Materials, vol. 7288. SPIE, 2009 